\title{
The Information Structure of Indulgent Consensus
}

\author{
Rachid Guerraoui, Member, IEEE, and Michel Raynal
}

\begin{abstract}
To solve consensus, distributed systems have to be equipped with oracles such as a failure detector, a leader capability, or a random number generator. For each oracle, various consensus algorithms have been devised. Some of these algorithms are indulgent toward their oracle in the sense that they never violate consensus safety, no matter how the underlying oracle behaves. This paper presents a simple and generic indulgent consensus algorithm that can be instantiated with any specific oracle and be as efficient as any ad hoc consensus algorithm initially devised with that oracle in mind. The key to combining genericity and efficiency is to factor out the information structure of indulgent consensus executions within a new distributed abstraction, which we call "Lambda." Interestingly, identifying this information structure also promotes a fine-grained study of the inherent complexity of indulgent consensus. We show that instantiations of our generic algorithm with specific oracles, or combinations of them, match lower bounds on oracle-efficiency, zero-degradation, and one-step-decision. We show, however, that no leader or failure detector-based consensus algorithm can be, at the same time, zero-degrading and configuration-efficient. Moreover, we show that leader-based consensus algorithms that are oracle-efficient are inherently zero-degrading, but some failure detector-based consensus algorithms can be both oracle-efficient and configuration-efficient. These results highlight some of the fundamental trade offs underlying each oracle.
\end{abstract}

Index Terms-Asynchronous distributed system, consensus, crash failure, fault tolerance, indulgent algorithm, information structure, leader oracle, modularity, random oracle, unreliable failure detector.

\section{INTRODUCTION}

\subsection{Context}

TnDERSTANDING the deep structure and the basic design principles of algorithms solving fundamental distributed computing problems is an important and challenging task. This task has been undertaken for basic problems such as distributed mutual exclusion [17], [30] and distributed deadlock detection [6], [20]. Another such basic problem is consensus [2], [13], [23]. This problem consists, for a set of $n$ processes, to propose each an initial value and, eventually, agree on one of the proposed values, even if some of the processes fail by crashing. Consensus is at the heart of reliable distributed computing and it is tempting to seek the fundamental structure of its algorithms, in particular, consensus algorithms that are optimal in terms of resilience and performance.

\subsection{Resilience Optimality}

Given that it is impossible to solve consensus deterministically in the presence of crash failures in a purely asynchronous system [13], several proposals have been made to augment the system with oracles that circumvent the impossibility. A first approach consists of introducing a random oracle [3] allowing us to design consensus algorithms that provide eventual decision with probability 1 . Another approach considers a failure detector oracle [7]

- R. Guerraoui is with LPD-IEC-EPFL, CH 1015 Lausanne, Switzerland. E-mail: rachid.guerraoui@epfl.ch.

- M. Raynal is with IRISA, Campus de Beaulieu, 35042 Rennes Cedex, France. E-mail: Michel.Raynal@irisa.fr.

Manuscript received 13 Mar. 2003; revised 2 July 2003; accepted 10 July 2003.

For information on obtaining reprints of this article, please send e-mail to: tc@computer.org, and reference IEEECS Log Number 118448. encapsulating eventual synchrony assumptions [12]. In particular, failure detector $\diamond \mathcal{S}$ has received a lot of attention. It provides each process with a list of processes suspected to have crashed, in such a way that every process that crashes is eventually suspected (completeness property) and there is a time after which some correct process is no longer suspected (accuracy property). Another approach consists of equipping the system with a leader oracle [21]. This oracle, denoted $\Omega$ in [8], provides the processes with a function leader which eventually always delivers the same correct process identity to all processes.

Oracles $\diamond \mathcal{S}$ and $\Omega$ are, in a precise sense, minimal in that each provides a necessary and sufficient amount of information about failures to solve consensus with a deterministic algorithm. Oracles $\diamond \mathcal{S}$ and $\Omega$ actually have the same computational power [8], [10]. The random oracle solves a nondeterministic variant of consensus [3] and is, strictly speaking, incomparable with the other two. This oracle is, however, in the sense of [9], as strong as consensus and, hence, also somehow minimal. Interestingly, the algorithms that rely on any of those oracles have all the common inherent flavor that consensus safety is never violated, no matter how the oracle behaves: They are indulgent toward their oracle [15]. In other words, the oracles are only necessary for the liveness property of consensus. A price to pay for this indulgence is that the upper bound $f$ on the number of processes that are allowed to crash has to be smaller than $n / 2$ (where $n$ is the total number of processes) and this is needed for each of these oracles [3], [7], [8].

\subsection{Performance Optimality}

This paper focuses on the performance of indulgent consensus algorithms in terms of time complexity (i.e., 
latency measured in terms of communication steps). That is, we consider the number of communication steps needed for the processes to reach a decision in certain runs of the execution. We overview here different optimality metrics along this direction and we define them precisely later in the paper.

- Oracle-efficiency. When an oracle behaves perfectly, the consensus decision can typically be expedited. More precisely, for all oracles discussed above, two communication steps are necessary and sufficient to reach consensus in failure-free runs where the oracle behaves perfectly, e.g., [19]. Algorithms that match this lower bound (e.g., [18], [26], [31]) are said to be oracle-efficient in the sense that they are optimized for the good behavior of the oracle.

- Zero-degradation. This property extends oracleefficiency from failure-free runs to runs with initial crashes [11]. Algorithms that have this property also match the two communication steps lower bound in runs with initial crashes. For instance, the consensus algorithms of [11] need only two communication steps to reach consensus when the oracle behaves perfectly, even if some processes had crashed initially. This is particularly important because consensus is typically used in a repeated form and a process failure during one consensus instance appears as an initial failure in a subsequent consensus instance. In a zero-degrading algorithm, a failure in a given instance does not impact the performance of any future instances.

- One-step-decision. If the processes exploit an initial knowledge on a privileged value or on a specific subset of processes, they can even, sometimes, reach consensus in a single communication step [5], e.g., when all noncrashed processes propose that privileged value. This can, for instance, be very useful if that specific value has a reasonable chance of being proposed more often than others. Algorithms that exploit such a knowledge to expedite a decision are called here one-step-decision algorithms.

- Configuration-efficiency. Finally, when all processes propose the same initial value, no underlying oracle is actually necessary to obtain a decision. In that case, two communication steps are also necessary and sufficient to reach a consensus decision, no matter how the underlying oracle behaves. Algorithms that match this lower bound are said to be configuration-efficient. (Such algorithms actually follow the condition-based approach introduced and investigated in [24].)

\subsection{Motivation}

In short, solving consensus goes through equipping distributed systems with additional oracles such as a failure detector, a leader oracle, or a random number generator. Interestingly, algorithms relying on such oracles are all indulgent, but they all require a correct majority. They do also have some inherent performance lower bounds in terms of time complexity. The objective of this work is to come up with a simple unified indulgent consensus algorithm that is generic and efficient. Genericity means here that we could easily instantiate the algorithm with any oracle, whereas efficiency means that the resulting algorithm should be as efficient as any ad hoc consensus algorithm designed for that specific oracle.

The first difficulty underlying this objective lies in factoring out the appropriate information structure that is common to efficient indulgent consensus algorithms, each of which might be using a different oracle and making use of specific algorithmic techniques. In fact, it is not entirely clear whether such a common structure could be precisely defined and whether the same generic algorithm could encompass the specific characteristics of a random oracle, a failure detector, and a leader oracle. The second difficulty has to do with the possible conflicting nature of the lower bounds. We know of no algorithm that matches all lower bounds recalled above and it is not clear whether such an algorithm can indeed be devised.

\subsection{Related Work}

In [18], several consensus algorithms were unified within the same framework, all, however, relying on $\diamond \mathcal{S}$-like failure detectors. A similar unification was proposed in [4], for $\Omega$-based consensus algorithms. In [1], [28], consensus algorithms that make use of several oracles at the same time were presented. In these hybrid algorithms, however, efficiency was not the issue and the oracles are used in a hardwired manner, e.g., they cannot be interchangeable. A first attempt to build a common consensus framework, unifying a leader oracle, a random oracle, and a failure detector oracle, was proposed in [25]. Unfortunately, algorithms derived by instantiating that framework with a given oracle are clearly not as efficient as ad hoc algorithms devised directly with that oracle. Efficient indulgent consensus algorithms were presented in [11]. However, for each oracle, a specific consensus algorithm is given.

\subsection{Contribution}

This paper factors out the information structure of efficient indulgent consensus algorithms within a new distributed abstraction, which we call Lambda. This abstraction encapsulates the use of any oracle (random, leader, or failure detector) during every individual round of indulgent consensus executions.

Using this abstraction, we construct a generic indulgent consensus algorithm that can be instantiated with any oracle, while assuming the highest number of possible failures, i.e., $f<n / 2$, and be as efficient as any ad hoc algorithm initially devised with that oracle in mind. The generic algorithm and the Lambda abstraction are constructed as two pluggable coroutines, with the round number of the consensus execution acting as the actual glue. Interestingly, the generic algorithm also enables the composition of different oracles, in an interchangeable way, generalizing the idea of hybrid consensus algorithms [1], [28].

The Lambda abstraction is defined by a set of precise properties that can be ensured in different ways according to the underlying oracle. The proposed generic consensus algorithm has a simple structure and its proof relies only on the properties of Lambda. As a convenient consequence, for any instantiation of the generic algorithm, it is sufficient to 
prove only that the particular oracle (or combination of oracles) ensures the properties defining Lambda. The genericity of the approach promotes a fine-grained composition of consensus optimizations. In particular, specific instantiations of Lambda have the novel, and noteworthy, feature of leading to indulgent consensus algorithms that are, at the same time, oracle-efficient, zero-degrading, and one-step-deciding.

Through new impossibility results on indulgent consensus, we also show that 1) no leader-based or failure detector-based consensus algorithm can be, at the same time, zero-degrading and configuration-efficient and 2) any leader-based consensus algorithm that is oracle-efficient is also zero-degrading (hence, such an algorithm cannot be configuration-efficient). We furthermore exhibit failure detector-based instantiations of our generic algorithm that are, at the same time, oracle-efficient and configurationefficient (hence, such an algorithm cannot be zero-degrading). These results highlight some of the fundamental trade offs underlying each oracle.

To summarize, the contributions of this paper are:

- A new distributed programming abstraction, called Lambda, which captures the information structure of indulgent consensus algorithms. This abstraction promotes genericity without hampering efficiency.

- New impossibility results on the composability of consensus optimality properties. We show that some time complexity lower bounds on consensus cannot all be matched with the same algorithm.

\subsection{Road-Map}

The paper consists of seven sections. Section 2 presents the computation model. Section 3 recalls the consensus problem and its oracles. Section 4 describes our generic algorithm and gives the specification of the Lambda abstraction. Section 5 provides particular instances implementing Lambda, each with a specific oracle, and discusses the efficiency of the resulting algorithms. Section 6 gives our impossibility results. Finally, Section 7 concludes the paper with some final remarks.

\section{Distributed Computation Model}

\subsection{Processes}

The computation model we consider is basically the asynchronous system model of [2], [7], [13], [23]. The system consists of a finite set $\Pi$ of $n>1$ processes: $\Pi=\left\{p_{1}, \ldots, p_{n}\right\}$. A process can fail by crashing, i.e., by prematurely halting. Until it possibly crashes, the process behaves according to its specification. If it crashes, the process never executes any other action. By definition, a faulty process is a process that crashes and a correct process is a process that never crashes. Let $f$ denote the maximum number of processes that may crash. We assume $f<n / 2$ (i.e., a majority of processes are correct).

\subsection{Channels}

Processes communicate and synchronize by sending and receiving messages through channels. Channels are assumed to be reliable: Messages are not altered or duplicated and any message sent by a correct process to a correct process is eventually received. There is no assumption about the relative speed of processes or on message transfer delays.

Our generic algorithm makes use of two communication abstractions that can be built on top of those channels: (simple) Broadcast and Reliable Broadcast abstractions [16]. Implementations of these communication abstractions using reliable channels are straightforward and described in [16], [29]. We simply recall their properties below.

The first abstraction is defined by two primitives: Broadcast and Delivery, the semantics of which are expressed by three properties, namely, validity, integrity, and termination. When a process $p$ executes $\operatorname{Broadcast}(m)$ (resp. Delivery $(m)$ ), we say that $p$ Broadcasts $m$ (resp. Delivers $m$ ). We assume that the messages are uniquely identified.

- Validity: If a process Delivers $m$, then some process has Broadcast $m$. (No spurious messages.)

- Integrity: A process Delivers a message $m$ at most once. (No duplication.)

- Termination: If a correct process Broadcasts $m$, then all correct processes Deliver $m$. (No message Broadcast by a correct process is missed by any correct process.)

Reliable Broadcast is defined by two primitives: $R \_$Broadcast and $R$ _Delivery, the semantics of which are also expressed by the three properties, validity, integrity, and termination. The only difference with the Broadcast abstraction is the termination property. The latter is stated here as follows:

- Termination: If 1 ) a correct process R-broadcasts $m$ or if 2) a process R-delivers $m$, then all correct processes R-deliver $m$. (No message R-broadcast by a correct process or R-delivered by a process is missed by a correct process.)

When there is no ambiguity, we use the term receive to mean Deliver or R-Deliver.

\section{Consensus and Its Oracles}

\subsection{The Consensus Problem}

In the Consensus problem, every process $p_{i}$ is supposed to propose a value $v_{i}$ and the processes have to decide on the same value $v$ that has to be one of the proposed values. More precisely, the problem is defined by two safety properties (validity and uniform agreement) and a liveness property (termination) [7], [13]:

- Validity: If a process decides $v$, then $v$ was proposed by some process.

- Uniform Agreement: No two processes decide differently. ${ }^{1}$

- Termination: Every correct process eventually decides on some value.

1. We actually consider here the uniform variant of consensus. It is important to notice that this does not make any difference for indulgent algorithms with the nonuniform variant of consensus (which only requires that no two correct processes decide differently) [15]. 
For presentation simplicity, we consider only consensus in its binary form: 0 and 1 are the only values that can be proposed.

\subsection{Leader Oracle}

A leader oracle is a distributed entity that provides the processes with a function leader() that returns a process name each time it is invoked. A unique correct leader is eventually elected, but there is no knowledge of when the leader is elected. Several leaders can coexist during an arbitrarily long period of time, and there is no way for the processes to learn when this "anarchy" period is over. The leader oracle, denoted $\Omega$, satisfies the following property: ${ }^{2}$

- Eventual Leadership: There is a time $t$ and a correct process $p$ such that, after $t$, every invocation of leader() by any correct process returns $p$.

We say that the oracle $\Omega$ is perfect if, from the very beginning, it provides the processes with the same correct leader.

$\Omega$-based consensus algorithms are described in [4], [11], [21], [27]. These algorithms are (or can be easily made) oracle-efficient: They can reach consensus in two communication steps when no process crashes and the oracle behaves perfectly. The $\Omega$-algorithm of [11] is not only oracleefficient, but also zero-degrading: It reaches consensus in two communication steps when the oracle is perfect and no process crashes during the consensus execution, even if some processes had initially crashed. The notion of zerodegradation means here that a failure in one consensus instance does not impact the performance of future consensus instances (where the failure appears as an initial failure).

\subsection{Failure Detector Oracle}

A failure detector $\diamond \mathcal{S}$ is defined as follows [7]: Each process $p_{i}$ is provided with a set denoted by suspected $d_{i}$. If $p_{j} \in$ suspected $_{i}$, we say that " $p_{i}$ suspects $p_{j}$.".Failure detector $\diamond \mathcal{S}$ satisfies the following properties:

- $\quad$ Strong Completeness: Eventually, every process that crashes is permanently suspected by every correct process.

- Eventual Weak Accuracy: There is a time after which some correct process is never suspected by the correct processes.

A $\diamond \mathcal{S}$ oracle is perfect if it suspects exactly the processes that have crashed and, hence, behaves as a perfect failure detector. That is, in addition to strong completeness, the oracle never suspects any noncrashed process.

Several $\diamond \mathcal{S}$-based consensus algorithms have been proposed in [7], [11], [18], [26], [31]. The algorithms of [18], [26], [31] reach consensus in two communication steps when the oracle is perfect and no process crashes: They are oracleefficient. The algorithm of [11] is also zero-degrading.

2. This property refers to a notion of global time. This notion is not accessible to the processes.

\subsection{Random Oracle}

A random oracle provides each process $p_{i}$ with a function random that outputs a binary value randomly chosen. Basically, random() outputs 0 (resp. 1 ) with probability $1 / 2$.

A binary consensus algorithm based on such a random oracle is described in [3]. In the case where the processes which have not initially crashed have the same initial value, this algorithm reaches consensus in two communication steps. (Note that, in this case, the algorithm does not actually use the underlying random oracle. This situation does actually correspond to the notion of configurationefficiency investigated in Section 6.)

\section{A Generic Consensus Algorithm}

Our generic consensus algorithm is described in Fig. 1. As announced in the Introduction, its combination of genericity and efficiency lies in the use of an appropriate information structure, called Lambda. This abstraction exports a single function, itself denoted lambda(), and this function encapsulates the use of any underlying oracle. The algorithm borrows its skeleton from [26]. ${ }^{3}$

\subsection{Two Phases per Round}

A process $p_{i}$ starts a consensus execution by invoking function consensus $\left(v_{i}\right)$, where $v_{i}$ is the value proposed by $p_{i}$ for consensus. Function consensus() is made up of two concurrent tasks: $T 1$ (the main task) and $T 2$ (the decision dissemination task). The execution of statement return $(v)$ by any process $p_{i}$ terminates the consensus execution (as far as $p_{i}$ is concerned) and returns the decided value $v$ to $p_{i}$.

In their main task (i.e., T1), the processes proceed through consecutive asynchronous rounds. Each round is made up of two phases. During the first phase of a round, the processes strive to select the same value, called an estimate value. Then, the processes try to decide during the second phase. This occurs when they get the same value at the end of the selection phase.

Each process $p_{i}$ manages three local variables: $r_{i}$ (current round number), est $1_{i}$ (estimate of the decision value at the beginning of the first phase), and est $2_{i}$ (estimate of the decision value at the beginning of the second phase). The specific value $\perp$ denotes a default value (which cannot be proposed by the processes).

\subsubsection{First Phase (Selection)}

The aim of this phase (line 104) is to provide all processes with the same estimate $\left(e s t 2_{i}\right)$. When this occurs, a decision will be obtained during the second phase. To attain this goal, this phase is made up of a call to the function lambda (). For any process $p_{i}$, the function has two input parameters: a round number $r$ (an integer) and est $1_{i}$ representing either a possible consensus value (i.e., a binary value in our case) or the specific value $\perp$. The function returns as an output parameter $e s t 2_{i}$, representing either a possible consensus value or the specific value $\perp$. A fundamental property ensured by this function is the

3. More precisely, our algorithm has the same structure as the $\diamond \mathcal{S}$-based algorithm of [26], but differs in its first phase. This phase relies on the Lambda abstraction instead of the particular $\diamond \mathcal{S}$ failure detector. 


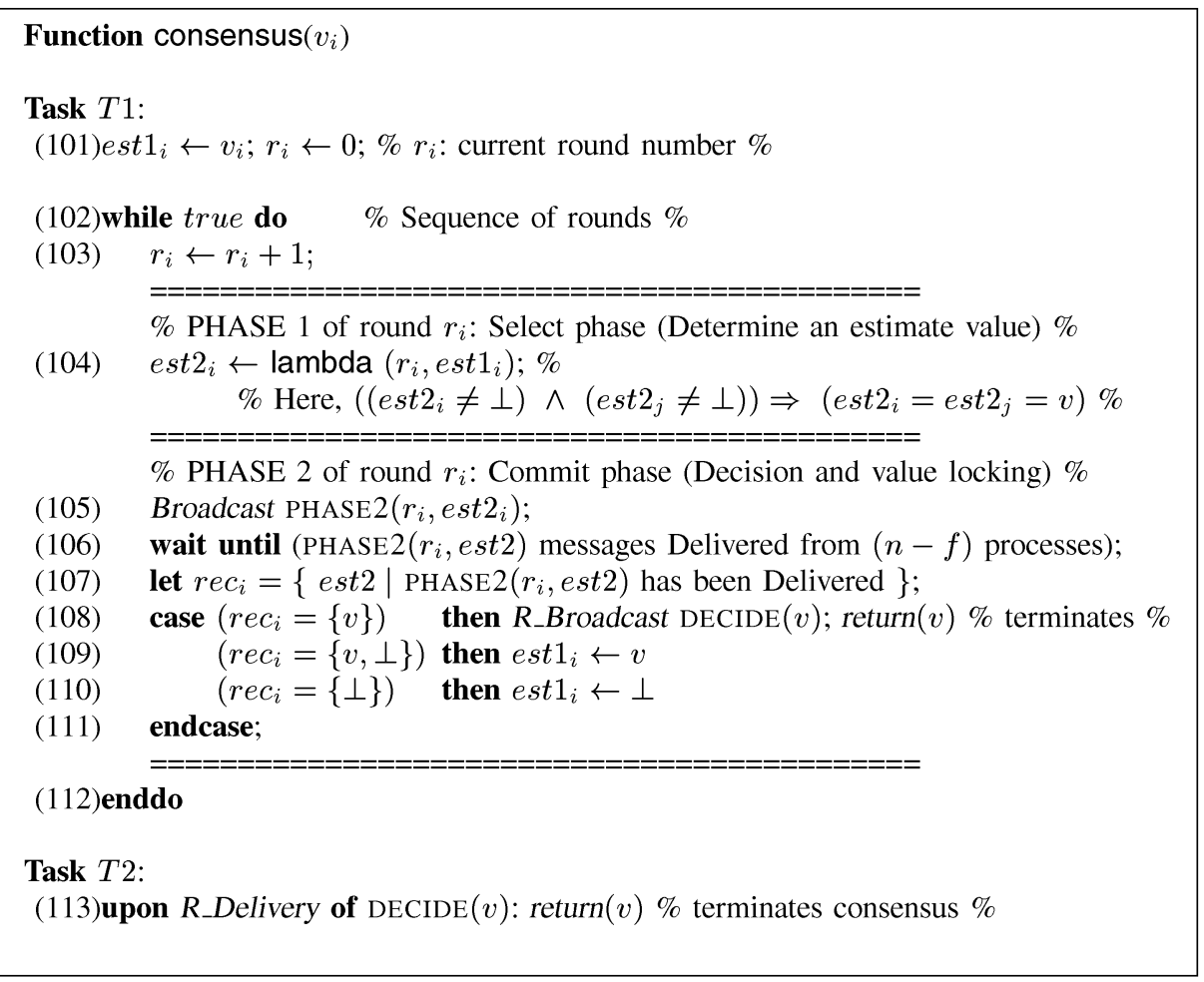

Fig. 1. The Generic Consensus Algorithm.

following: For any two processes, $p_{i}$ and $p_{j}$ that, during a round $r$, return from lambda $(r,-)$, est $2_{i}$ and $e s t 2_{j}$, respectively, we have:

$$
\left(\left(e s t 2_{i} \neq \perp\right) \wedge\left(e s t 2_{j} \neq \perp\right)\right) \Rightarrow\left(\text { est } 2_{i}=e s t 2_{j}=v\right) .
$$

\subsubsection{Second Phase (Commitment)}

The second phase (lines 105-111) starts with an exchange of the new estimates (note that these are equal to the same value $v$ or to $\perp$ ). Then, the behavior of a process $p_{i}$ depends on the set $r e c_{i}$ of estimate values it has gathered. There are three cases to consider [26].

1. If $r e c_{i}=\{v\}$, then $p_{i}$ decides on $v$. Note that, in this case, as $p_{i}$ receives $v$ from $(n-f)>n / 2$ processes, any process $p_{j}$ receives $v$ from at least one process. (Obviously, the algorithm remains correct if a process waits for $y$ messages, with $n / 2<y \leq n-f$.)

2. If $\operatorname{rec}_{i}=\{v, \perp\}$, then $p_{i}$ considers $v$ as its new estimate value (this is because some process might have decided $v$ ) and proceeds to the next round.

3. if $\operatorname{rec}_{i}=\{\perp\}$, then $p_{i}$ adopts $\perp$ as estimate and proceeds to the next round. The adoption of $\perp$ as estimate is transitory. (An estimate equal to $\perp$ will be updated to a non- $\perp$ value by the lambda() function called at the next round.)

It is important to notice that, at any round, lines 108 and 110 are mutually exclusive: If some process executes one of them, then no process can execute the other. This exclusion actually "locks" the decided value, thereby guaranteeing consensus agreement. It follows that, when the processes start a new round $r>1$, est $1_{i}$ variables whose values are different from $\perp$ are equal to the same value $v$ : The value is indeed locked.

The use of Reliable Broadcast (line 108) has the following motivation: Given that any process that decides stops participating in the sequence of rounds and all processes do not necessarily decide during the same round, it is possible that processes that proceed to round $r+1$ wait forever for messages from processes that have terminated at $r$. By disseminating the decided value, the Reliable Broadcast primitive prevents such occurrences.

\subsection{The Lambda Abstraction}

This section defines the properties of our Lambda abstraction. It states the properties any infinite sequence of invocations of lambda () function has to satisfy. Section 5 will then show how these properties can be ensured using various underlying oracles.

- Validity: No process invokes lambda $(1, \perp)$. Moreover, if $p_{i}$ returns $a \neq \perp$ from lambda $(r,-)$, then some process invoked lambda $\left(r^{\prime}, a\right)$ with $r^{\prime} \leq r$.

- Quasi-agreement: Let $p_{i}$ and $p_{j}$ be any two processes that invoke lambda $(r,-)$ and get the values $a$ and $b$, respectively. If $(a \neq \perp)$ and $(b \neq \perp)$ then $a=b$.

- $\quad$ Fixed point: For any round number $r$, if all processes that invoke lambda $(r,-)$ invoke it with the same value $a$ (i.e., lambda $(r, a)$ ), then $a$ and $\perp$ are the only values that can be returned by any invocation of lambda $\left(r^{\prime},-\right), \forall r^{\prime} \geq r .^{4}$

4. Using the terminology of [7], this property means that the value $v$ is locked: No different value $v^{\prime}$ can be decided. Using the terminology of [13], the configuration is monovalent as only $v$ can be decided. 
- Termination: For any round $r$, if all correct processes invoke lambda $(r,-)$, then all correct processes return from the invocation.

- Eventual convergence: If all correct processes keep on repeatedly invoking lambda () with increasing round numbers, then there is a round $r$ such that lambda $(r,-)$ returns the same non- $\perp$ value to all correct processes.

\subsection{Correctness of the Generic Algorithm}

Assuming $f<n / 2$ and the Lambda abstraction, this section discusses how the algorithm of Fig. 1 satisfies the validity, uniform agreement and termination properties of consensus.

Theorem 1 (Validity). Any decided value is a proposed value.

Proof. The specific value $\perp$ cannot be decided (line 108). By the validity of the Lambda abstraction as well as the integrity and validity properties of the Broadcast primitives, the est $1_{i}$ and $e s t 2_{i}$ variables can only contain proposed values or $\perp$.

Theorem 2 (Uniform Agreement). No two processes decide different values.

Proof. This follows from the quasi-agreement and fixed point properties of the Lambda abstraction as well as the integrity and validity properties of the broadcast primitives.

Let $r$ be the smallest round during which some process decides ("decide $v$ during $r$ " means "during $r$, execute line 108 with $\left.r e c_{i}=\{v\}^{\prime \prime}\right)$. We first show that 1) the processes that decide during $r$ decide $v$ and 2) all estimates are equal to $v$ at the end of $r$. We then show from 2) that no other value can be decided in a subsequent round.

At the end of the first phase of $r$ (just after line 104 and before line 105), we have

$$
\left(\left(\text { est } 2_{i} \neq \perp\right) \wedge\left(\text { est } 2_{j} \neq \perp\right)\right) \Rightarrow\left(\text { est } 2_{i}=e s t 2_{j}=v\right) .
$$

This follows from the quasi-agreement property of the Lambda abstraction. As $\perp$ cannot be decided, it follows that, if two processes decide during $r$, they decide the same non- $\perp$ value at line 108 .

Assuming that some process $p_{i}$ decides $v$ during $r$, we now prove that the estimate $e s t 1_{j}$ of any process $p_{j}$ that progresses to $r+1$ is equal to $v$ at the end of $r$. As there are more than $n / 2$ PHASE2 messages carrying the same $v$ (these are the messages that allowed $p_{i}$ to decide $v$ during $r$ ), then, by the integrity and validity properties of the Broadcast primitive, $p_{j}$ must have Delivered at least one of those PHASE2 $(r, v)$ messages. Consequently, $p_{j}$ executed line 109 and updated $e s t 1_{j}$ to $v$. It follows that all the processes that start $r+1$ have their estimate variables equal to $v$.

Consider now round $r+1$. As the estimates of the processes that start $r+1$ are equal to the same non- $\perp$ value, namely $v$, it follows from the fixed point property of the Lambda abstraction that no value different from $v$ can be decided in a future round.

Lemma 1. No correct process blocks forever in a round.
Proof. This follows from 1) $f$ being the maximum number of processes that can crash, 2) the termination properties of Lambda, as well as 3) the termination and integrity properties of the Broadcast primitives. We show this more precisely below.

If a process decides, then, by the termination property of the Reliable Broadcast of the DECIDE message, all correct processes decide. Hence, no correct process blocks forever in a round. Assume by contradiction that no process decides. Let $r$ be the smallest round number in which some correct process $p_{i}$ blocks forever. So, $p_{i}$ blocks at line 104 or 106 . By the termination property of Lambda, no correct process blocks forever at line 104. Consider now the case of line 106: The fact that $p_{i}$ cannot block follows directly from the assumption that there are at most $f$ crashed processes, from which we conclude that at least $(n-f)$ processes Broadcast the corresponding messages: The integrity and termination properties of the Broadcast lead to a contradiction.

Theorem 3 (Termination). Every correct process eventually decides.

Proof. This follows from

1. Lemma 1,

2. $f$ being the maximum number of processes that can crash,

3. the termination and convergence properties of Lambda, as well as

4. the integrity and termination properties of the Broadcast primitives.

The proof is by contradiction. Assume that no process decides. By Lemma 1, the correct processes progress from round to round. Hence, by the eventual convergence property of Lambda, there is a round $r$ during which all processes have the same value $v$ at the end of their first phase. It follows that the PHASE2 $(r,-)$ messages carry the same value $v$. By the integrity and termination properties of the Broadcast, for any process $p_{i}$ executing the second phase of $r$, we have $\operatorname{rec}_{i}=\{v\}$, from which we conclude that the correct processes decide at line 108 .

\section{Oracle-Based Implementations of LamBda}

This section describes modules that implement the Lambda abstraction using various forms of oracles, namely, a leader oracle, a failure detector oracle, and a random oracle. A module provides a piece of code implementing the function lambda $\left(r_{i}, e s t 1_{i}\right)$ invoked by a process $p_{i}$ and returning a value in $e s t 2_{i}$. All these implementations use a local variable per process $p_{i}$, denoted prev_est $1_{i}$. The aim of this local variable is to ensure the fixed point property by keeping the last non- $\perp$ value of $e s t 1_{i}$ over subsequent invocations of the lambda() function.

As observed in the Introduction, the generic consensus algorithm and the Lambda abstraction act as two software components pluggable together. More precisely, from the point of view of each process $p_{i}$, an execution of the generic algorithm together with an implementation of the Lambda abstraction is the conjunction of two "co-routines" that 


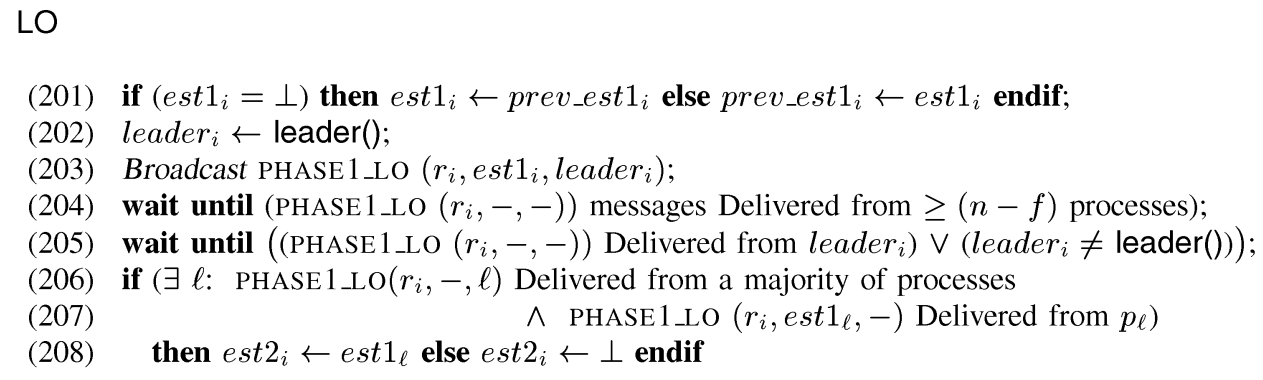

Fig. 2. $\Omega$-based module.

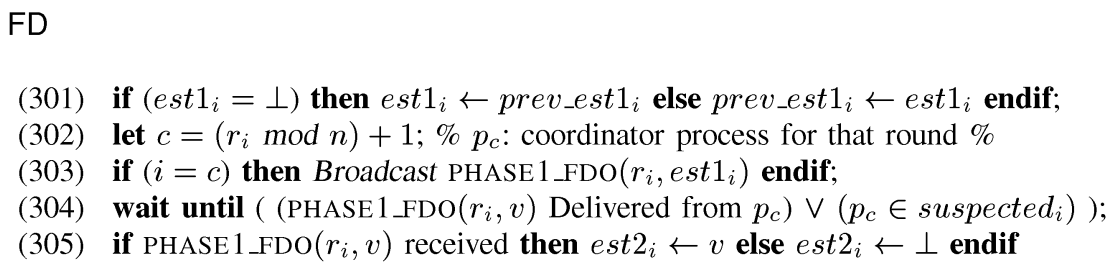

Fig. 3. $\diamond$ cal $S$-based module.

progress in turn. During each round, the control flow at $p_{i}$ moves during the first phase from the main co-routine (i.e., the algorithm), to the co-routine implementing Lambda, and then returns to the main co-routine for the second phase before progressing to the next round.

\subsection{Leader Module}

An implementation of lambda $\left(r_{i}\right.$, est $\left._{i}\right)$ based on $\Omega$ is described in Fig. 2. After resetting $e s t 1_{i}$ to its last non- $\perp$ value (if $e s t 1_{i}=\perp$ ), every process $p_{i}$ first invokes the oracle $\Omega$. The latter provides $p_{i}$ with the identity of some process (i.e., the name of a leader-line 202). Then, $p_{i}$ exchanges with all other processes its current estimate value plus the name of the process it considers leader (line 203). When $p_{i}$ has got such messages from at least $(n-f)$ processes (line 204), $p_{i}$ checks if some process $p_{\ell}$ is considered leader by a majority of the processes. If there is such a process $p_{\ell}$, and $p_{i}$ has got its current estimate value est $1_{\ell}$, then $p_{i}$ considers $e s t 1_{\ell}$ as the value of est $2_{i}$. In the other cases, $p_{i}$ sets $e s t 2_{i}$ to $\perp$ (lines 206-208).

Theorem 4. The algorithm of Fig. 2 implements Lambda using $\Omega$.

Proof. ${ }^{5}$ We have to show that the LO module, described in

Fig. 2, satisfies the validity, quasi-agreement, fixed point, termination, and eventual convergence properties defined in Section 4.2.

Validity and termination follow directly from the algorithm and $f$ being the maximum number of processes that can crash. Quasi-agreement follows from the fact that an $e s t 2_{i}$ variable is equal to $\perp$ or the $e s t 1_{\ell}$ value of a process $p_{\ell}$ (let us notice that there is at most one process $p_{\ell}$ that is considered leader by a majority of processes). The fixed point property follows from the fact

5. Line 205 is related to the zero-degradation property (see the discussion below). It concerns neither the safety nor the liveness of the Lambda abstraction and is consequently not used in this proof. that, if all $e s t 1_{i}$ are equal to some value $v$, then only $v$ or $\perp$ can be output at line 208 (notice that the second phase of the consensus algorithm can set est $1_{i}$ only to $v$ or $\perp$ ). Therefore, if all est $1_{i}$ are equal to $v$ at the beginning of $r$, due to the management of the prev_est $1_{i}$ variables, all processes (that have a value) will have the same value $v$ after executing line 201 during the next round. The eventual convergence property follows from the fact that there is a time after which all processes have the same correct leader $p_{\ell}$; when this occurs, $p_{\ell}$ imposes its estimate to all processes.

The generic consensus algorithm, instantiated with such an implementation of the function lambda (), boils down to the $\Omega$ algorithm of [11] which, as we pointed out, is the most efficient $\Omega$-based algorithm we know of. When $\Omega$ is perfect, it provides the processes with the same correct process as a leader from the very beginning. Moreover, due to line 205, the test of lines 206-207 is then satisfied as $p_{i}$ has got the estimate value from the common leader $p_{\ell}$ (which is leader $_{i}$ ). It follows that, at line 208, est $2_{i}$ is set to the value of $e s t 1_{\ell}$ (which is different from $\perp$ ). Thus, it is easy to see that, in this case, all processes get the same estimate value after the execution of lambda $(1,-)$ and the protocol terminates in two communication steps despite initial process crashes. So, the algorithm is zero-degrading (hence, also oracle-efficient).

\subsection{Failure Detector Module}

A $\diamond \mathcal{S}$-based implementation of lambda $\left(r_{i}, e s t_{i}\right)$ is described in Fig. 3. Its principle is particularly simple. Each round has a coordinator (line 302) that tries to impose its estimate value to all the processes (line 303). As the coordinator can crash, a process relies on the strong completeness property of failure detector $\diamond \mathcal{S}$ in order not to wait indefinitely (line 304). If a process $p_{i}$ gets a value $v$ from the round 


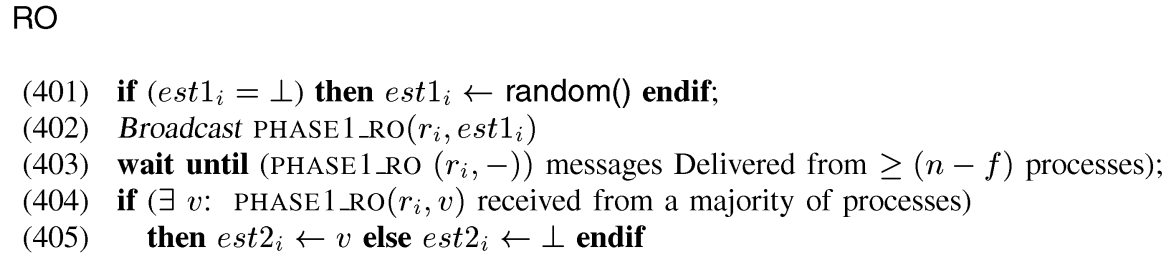

Fig. 4. Random-based module.

coordinator, $p_{i}$ sets $e s t 2_{i}$ to $v$. If $p_{i}$ suspects the current round coordinator to have crashed, $p_{i}$ sets est $2_{i}$ to $\perp$ (line 305). In order not to miss the correct process that is eventually no longer suspected (eventual weak accuracy), all processes have to be considered in turn as coordinator until a value is decided. This is realized with the help of the mod function at line 302.

Theorem 5. The algorithm of Fig. 3 implements Lambda using $\diamond \mathcal{S}$.

Proof. The proof is similar to the one used for Theorem 4. $\square$

The generic consensus algorithm instantiated with such an implementation of the Lambda abstraction boils down to the $\diamond \mathcal{S}$-based consensus algorithm described in [26]. It is easy to see that the resulting algorithm needs only two communication steps to decide when the first coordinator is correct and the $\diamond \mathcal{S}$ failure detector is perfect. So, this algorithm is oracle-efficient. However, it is not zerodegrading because, if the first coordinator has crashed, the algorithm has to proceed to the second round. In this case, at least three communication steps are required to decide. (One to proceed from the first to the second round and the others in the second round.) A simple way to get a zero-degrading $\diamond \mathcal{S}$-based consensus algorithm, despite the crash of the first coordinator, consists of customizing its first round. More precisely, the lambda () function is then implemented as follows:

- Round $r=1$ : This round uses a module similar to the one described in Fig. 2 except for its line 202 (the line where the $\Omega$ oracle is used) which is replaced by the following statement:

$$
\text { leader }_{i} \leftarrow \min \left(\Pi-\text { suspected }_{i}\right) .
$$

- Round $r>1$ : These rounds use the module described in Fig. 3.

When the failure detector is perfect, all processes get the same correct process as the "leader" of the first round, do not suspect it, and, consequently, the decision is obtained during that round. When we consider this implementation of the lambda () function, the first round satisfies validity, quasi-agreement, fixed point, and termination, whereas the other rounds additionally satisfy eventual convergence.

\subsection{Random Module}

A random-based implementation of Lambda is described in Fig. 4 . When a process starts a new round with $e s t 1_{i}=\perp$, it sets $e s t 1_{i}$ randomly to 0 or 1 . The processes then exchange their current estimates values and each process $p_{i}$ looks for a value that is a majority value. If such a value is obtained, process $p_{i}$ assigns it to $e s t 2_{i}$. If $p_{i}$ does not see a majority estimate value, $p_{i}$ sets $e s t 2_{i}$ to $\perp$. Note that, if est $1_{i}=\perp$ at the beginning of a round, process $p_{i}$ can conclude that both values have been proposed. Note also that, at the beginning of the first round, no estimate value is equal to $\perp$.

Theorem 6. Using random, the algorithm of Fig. 4 implements the validity, quasi-agreement, and termination properties of Lambda.

Proof. Straightforward from the algorithm.

The randomized consensus algorithm obtained when using this implementation of the Lambda abstraction boils down to the algorithm proposed in [3]. As noticed in Section 3.4, in the particular case where the processes that have not initially crashed propose the same initial value, this algorithm does not use the underlying random oracle and reaches consensus in two communication steps. Actually, this algorithm uses the random oracle to allow the processes to "eventually" start a round with the same estimate value. When that round is reached, the process can decide without the help of the oracle. The algorithm satisfies the eventual convergence property with probability 1 .

\subsection{Module Composition}

Interestingly, it is possible to provide implementations of the Lambda abstraction based on combinations of the previous LO, FD, and RO modules (or even any $X Y$ module satisfying the validity, quasi-agreement, fixed point, termination, and eventual convergence properties of the Lambda abstraction). Such combinations provide hybrid consensus algorithms that generalize the specific combinations that have been proposed so far (namely, the algorithms that combine a failure detector oracle with a random oracle [1], [28]).

As examples, let us consider two module combinations that merge the LO and FD modules.

- The first combination is the LO_FD_1 module defined as follows:

- The odd rounds of lambda() are implemented with the LO module (Fig. 2),

- The even rounds are implemented with the FD module (Fig. 3) where the coordinator $p_{c}$ is defined as follows: $c=\left(\left(r_{i} / 2\right) \bmod n\right)+1 .^{6}($ Note

6. In that way, no process is a priori prevented from being the correct process that eventually is not suspected by the other processes. 


$$
\begin{aligned}
& \text { PV } \\
& \text { (501) if ( PHASE1_LO }\left(r_{i}, \alpha,-\right) \text { Delivered from a majority of processes including } p_{\ell} \text { ) } \\
& \text { (502) then } R_{-} \text {Broadcast } \operatorname{DECIDE}(\alpha) ; \operatorname{return}(\alpha) \% \text { terminates } \% \\
& \text { (503) else if (PHASE } 1_{-} \mathrm{LO}\left(r_{i}, \alpha,-\right) \text { has been Delivered) then prev_est } 1_{i} \leftarrow \alpha \text { endif } \\
& \text { (504) endif }
\end{aligned}
$$

Fig. 5. Privileged value module.

$$
\begin{aligned}
& \text { PS } \\
& \begin{array}{l}
(601) \quad \text { if }\left(\forall p_{j} \in S: \quad \text { PHASE1_LO }\left(r_{i}, v,-\right) \text { Delivered from } p_{j} \wedge p_{\ell} \in S\right) \\
\% \text { all processes of } S \text { have sent the same value } v \% \\
\text { then } R \_B r o a d c a s t \operatorname{DECIDE}(v) ; \text { return }(v) \% \text { terminates } \% \\
(602) \quad \text { else let } x \text { be the estimate Delivered from a } p_{j} \in S ; \text { prev_est } 1_{i} \leftarrow x \\
(603) \text { endif }
\end{array}
\end{aligned}
$$

Fig. 6. Privileged set of processes module.

that this composition requires a slight modification of the round coordinator definition.)

- The second combination is the LO_FD_2 module defined as follows. Each round of lambda() is implemented by the the concatenation made up of the LO module immediately followed by the FD module.

It is easy to see that each of the resulting LO_FD_1 and LO_FD_2 modules satisfies the properties associated with the Lambda abstraction. Other combinations could be defined in a similar way. Such combinations have to be such that, given a round $r$, all processes use the same module composition during $r$.

Appropriate combinations merging the $\mathrm{RO}$ module to the LO and FD modules, provide implementations of Lambda that satisfies its validity, quasi-agreement, fixed point, and termination properties. As far as the eventual convergence property is concerned, it is satisfied if the LO (or FD) module is involved in an infinite number of rounds. In the other cases, it is only satisfied with probability 1 (assuming RO is involved in an infinite number of rounds).

In that sense, the generic algorithm provides indulgent consensus algorithms that can benefit from the best of several "worlds" (leader oracle, failure detector oracle, or random oracle).

\subsection{One-Step Decision}

This section considers two additional assumptions that, when satisfied by an actual run, allow the consensus algorithm to expedite the decision, i.e., to terminate in one communication step [5]. Each of these assumptions relies on a specific a priori agreement. More precisely, the first one assumes an a priori agreement on a particular value and allows a one-step decision when enough processes do propose that value. The other assumes that there is a predefined majority set of processes and allows a one-step decision when those processes do propose the same value.

Interestingly, these additional assumptions can be merged in any deterministic implementation of Lambda (i.e., LO, FD, or SV-defined in the next section). In the following, we illustrate this idea by combining a specific implementation with the $\Omega$-based implementation of Lambda given in Fig. 2. For a specific initial configuration, the processes can reach a decision in one communication step. Interestingly, the onestep decision characteristic of the resulting algorithm does not impact the performance of the algorithm when the initial configuration is not a specific one.

When combined with the LO module, each of the modules introduced below (named PV-Fig. 5-and PS -Fig. 6) assumes that there is a leader $p_{\ell}$ defined in the LO module (line 202 of Fig. 2). The merging of the LO module with the PV module (respectively, PS) concerns only the first round. This merging is achieved through the LO module of Fig. 2. More precisely, we test if there is a leader as defined in the LO module (line 202 of Fig. 2). In that case, we invoke the PV (respectively, PS) module.

\subsubsection{Existence of a Privileged Value}

Some applications have the property that some predetermined value $(\alpha)$ appears much more often than other values. This means that $\alpha$ is usually proposed much more often than other values. The a priori knowledge of such a predetermined value can help expedite the decision. This can be done by plugging the following module PV, described in Fig. 5, as described above. The idea underlying this module is the following: If there is a leader $p_{\ell}$ (Fig. 2, line 206) and a majority of processes including $p_{\ell}$ have their current estimates equal to $\alpha$, then $p_{i}$ decides $\alpha$ (line 502). Otherwise, if $p_{i}$ has Delivered a PHASE1_LO message carrying $\alpha$, then $p_{i}$ updates its prev_est $1_{i}$ local variable to $\alpha$. It is easy to see that, in any run where the processes that have not initially crashed propose $\alpha$ and the oracle is perfect, the processes decide in one communication step.

Theorem 7. The previous combination merging the LO and $P V$ modules provides a correct implementation of the Lambda abstraction. When used in the consensus protocol described in Fig. 1, it allows the processes to decide, in one communication step, when the privileged value $\alpha$ is the only proposed value and the oracle is perfect. 
Proof. Let us first notice that, if no process executes line 502, then the only difference between the LO+PV merging and LO lies in the fact that some processes possibly set their prev_est $1_{i}$ variables to $\alpha$ (which is then a proposed value). This does not modify the output of the lambda () function for that round.

Let us now consider the case where a process decides at line 502. In this case, the process decides $\alpha$, which is then the estimate value of the unique leader $p_{\ell}$ of that round. Moreover, as, in this case, $\alpha$ has been sent by a majority of processes and $f<n / 2$, it follows that all the processes that do not decide at line 502 execute line 503, updating prev_est $1_{i}$ to $\alpha$.

If a process decides during the second phase, it necessarily decides the current estimate of $p_{\ell}$, namely, $\alpha$. Assume some process $p_{i}$ does not decide during the second phase. There are two cases to consider. Process $p_{i}$ executes line 109 or line 110 (Fig. 1). If $p_{i}$ executes line 109, then $p_{i}$ sets $e s t 1_{i}$ to $\alpha$. If $p_{i}$ executes line 110 , then $p_{i}$ sets est $1_{i}$ to $\perp$, but then, at the beginning of the next round, $p_{i}$ will reset $e s t 1_{i}$ to prev_est $1_{i}$, whose value is now $\alpha$ (it has been set to that value at line 503). It follows that if a process decides at line 502,1 ) the processes that decide during the second phase of the round decide $\alpha$ and 2) the processes that start the next round have their estimates $e s t 1_{i}$ equal to $\alpha$ and, so, no other value can be decided in a later round.

Let us now consider the case where all the processes propose the privileged value $\alpha$. No matter which process $p_{\ell}$ is considered leader, $p_{i}$ receives $\alpha$ from a majority of processes including $p_{\ell}$ and, consequently, decides at line 502. So, in that case, the processes decide in one communication step.

\subsubsection{Existence of a Privileged Set of Processes}

This specific case considers the situations where there is a predetermined set $S$ of processes $(f<n / 2<|S|)$, initially known by each process. When processes in $S$ do not crash and propose the same value, it is possible to terminate in one communication step. The corresponding PV module is described in Fig. 6. It is merged with the LO as described previously. The proof that this combination is correct is similar to the previous one.

\subsubsection{Discussion}

When we look at the PV and PS modules, we can observe that they are dual in the following sense: PV is "value" oriented: It considers the case where the processes propose the same predetermined value. On the other hand, PS is "control" oriented: It considers the case where a predefined set of processes propose the same nonpredetermined value. In both cases, the improvement results from an a priori agreement, either on the value $\alpha$ or on the set $S$.

Let us notice that the introduction of module PV or module PS does not add any communication cost to the resulting consensus algorithm. Hence, defining a priori a privileged value $\alpha$ or a majority set of processes $S$ and trying to exploit it to expedite a decision is an overhead-free operation (whatever the value chosen or the set selected, it entails no additional communication cost).
Let us finally observe that, when the set of values does not allow the PV (or PS) module to terminate in one communication step, the consensus algorithm remains zerodegrading: It still terminates in two communication steps despite initial crashes if the underlying $(\Omega$ or $\diamond \mathcal{S})$ oracle behaves perfectly. So, combining the additional assumption that "there is a privileged value" or "there is a predefined majority set of processes" with the use of an $\Omega$ or $\downarrow \mathcal{S}$ oracle does not prevent zero-degradation. In a precise sense, onestep decision and zero-degradation are compatible. This has to be contrasted with the main result of the next section (Theorem 9) which shows that configuration-efficiency cannot be combined with zero-degradation.

\section{Configuration Efficiency}

As we have pointed out (Section 3.4 and Section 5.3), when all the processes (that have not initially crashed) propose the same initial value, no underlying oracle is necessary to obtain a decision and two communication steps are necessary and sufficient to get a decision. We call an algorithm that matches this lower bound each time the initial values are the same and no matter how the underlying oracle behaves a configuration-efficient algorithm.

This section first presents a simple module that, when used to implement the first round of the lambda() function (the other rounds being implemented with the LO, FD, or $\mathrm{RO}^{7}$ module, or a combination of them), provides a configuration-efficient consensus algorithm. Then, it is shown (Theorem 9) that no $\Omega$ or $\diamond \mathcal{S}$-based consensus protocol can be, at the same time, configuration-efficient and zero-degrading. In a precise sense, these optimization techniques are incompatible. On the contrary, and as we have seen, a random-based consensus algorithm can be at the same time configuration-efficient and zero-degrading when a single value is proposed (we have also seen that, in this case, the random oracle is not used). Finally, we show that it is possible to trade zero-degradation with configuration-efficiency in the case of $\diamond \mathcal{S}$ (in the sense that both cannot be simultaneously provided), but not in the case of $\Omega$ (Theorem 10).

\subsection{Same Value Module}

We present here a simple implementation of the lambda () function (Fig. 7) that is only based on the values proposed by the processes: No oracle is used. The processes exchange their current estimates values and look for a value that is a majority value. If such a value exists, process $p_{i}$ assigns it to est $2_{i}$. If a process $p_{i}$ does not see a majority estimate value, it sets $e s t 2_{i}$ to $\perp$. Except for its first line, this module, denoted by SV, is the same as the RO module: The only difference lies in the fact that SV does not use any underlying oracle. When used in the first round, SV and RO are actually the same module (this is because, when the first round starts, we have est $_{i} \neq \perp$ ).

Theorem 8. The algorithm of Fig. 7 ensures the validity, quasiagreement, fixed point, and termination properties of Lambda.

7. In the latter case, the eventual convergence property of Lambda can only be guaranteed with probability 1 . 


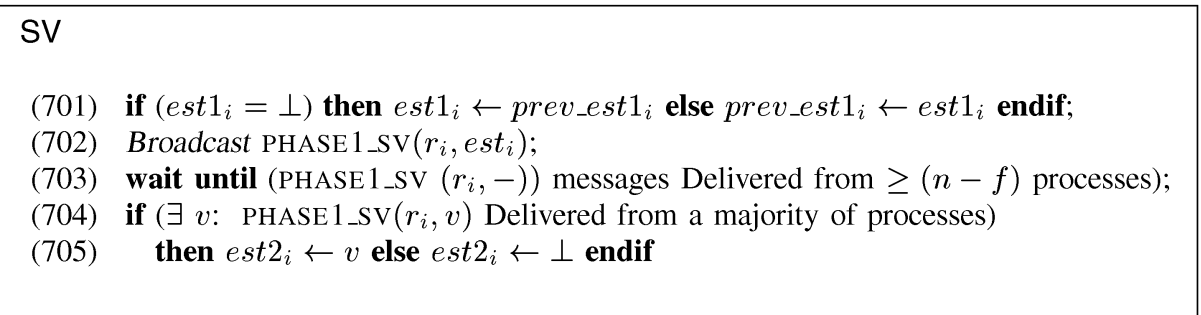

Fig. 7. Same value module.

Proof. Straightforward from the algorithm.

This implementation does not satisfy eventual convergence, so the termination of the consensus algorithm is not guaranteed in all cases. This implementation is particularly interesting when there is a high likelihood that the processes do propose the same value. In such cases, the resulting consensus algorithm is zero-degrading and terminates in two communication steps. Interestingly, this module can be used in combination with other oracle-based modules to provide Lambda implementations.

\subsubsection{Remark}

Assuming no more than $f$ processes can crash, the implementation of Lambda based on the SV (resp. RO, LO, and FD) module ensures the validity, quasi-agreement, fixed point, and termination properties of Lambda. SV does not ensure eventual convergence, while (due to their powerful underlying oracles) LO and FD ensure it. RO can be seen as at an intermediate level as it ensures eventual convergence only probabilistically. So, RO can be seen as SV enriched with a "relatively weak oracle" whose aim is to help obtain eventual convergence.

\subsection{Impossibility Result}

Assuming $f<n / 2$ and $A$ being any $\Omega$-based or $\diamond \mathcal{S}$-based consensus algorithm, we show here that $A$ cannot be simultaneously zero-degrading and configuration-efficient. The proof technique uses indistinguishability arguments, both 1) among runs without crashes and runs with crashes and 2) among runs where the oracle behaves perfectly and runs where the oracle does not.

As our impossibility result is a lower bound on a number of communication steps, it is stated and proven assuming a round-based full-information algorithm [14], i.e., we assume that processes exchange the maximum information they can exchange within every message. That is, whenever a process transmits a message, it transmits it to all and includes its current state. Processes proceed in rounds. In every round, a process sends a message to all processes. Before moving to the next round, the process waits for messages from a majority and, depending on the oracle, it waits for other messages. Basically, if the algorithm is based on $\Omega$, the process also waits for a message from the leader. If the algorithm is based on $\nabla \mathcal{S}$, the process also waits for a message from every nonsuspected process. Assuming this strong model strengthens our impossibility result.

As we defined it, the notion of zero-degradation means that the algorithm reaches consensus in 2 communication steps (rounds) in any run where no process crashes, except possibly initially, and the oracle behaves perfectly. Let us recall here that $\Omega$ behaves perfectly in a run if it always outputs the same correct process to all processes in that run; $\diamond \mathcal{S}$ behaves perfectly when every process that crashes is eventually suspected (in a permanent way) by all correct processes and no process is suspected before it crashes. Note that, when we say here that a run reaches consensus, we mean that all correct processes have decided.

Theorem 9. Assuming $f<n / 2$, no $\Omega$ or $\diamond \mathcal{S}$-based consensus algorithm can be zero-degrading and configuration-efficient.

Proof. We need to prove that if consensus can be reached in two rounds in any run of $A$ where the oracle behaves perfectly and no process crashes, except initially (i.e., $A$ is zero-degrading), then there exists a run of $A$ that does not reach consensus in two communication steps even if all processes have the same initial values (i.e., $A$ cannot be configuration-efficient).

The proof first considers the case of three processes (i.e., $n=3$ and $f=1$ ). Then, it considers the case $n>3 .^{8}$ Furthermore, we first consider a communication-closed model [14]: If a process does not deliver, in a round $r$, a message Broadcast to it in that round, the process never delivers that message. We shall later discuss the generalization of our proof argument for the communicationopen model.

Case $n=3$. We prove our result using simple indistinguishability arguments among four runs: $R_{1}-R_{4}$. We depict the important messages of these runs in Fig. 8. Messages that are Broadcast and not Delivered or sent by a process to itself are not indicated; the value proposed by a process is indicated inside square brackets "[ ]" and the value decided inside parentheses "( )".

1. At least until the second round, run $R_{1}$ is similar for processes $p_{2}$ and $p_{3}$ to a run where $p_{1}$ has initially crashed. Without loss of generality, if $A$ is zero-degrading, then $A$ must have a run such as run $R_{1}$. In this run, $\Omega$ would output the same leader process, say $p_{2}$, at all processes and $\diamond \mathcal{S}$ would output, say $p_{1}$, at all processes. In both cases, messages from $p_{1}$ are missed by $p_{2}$ and $p_{3}$ because they consider $p_{1}$ to have initially crashed: Messages received by $p_{1}$ are hence not relevant for our purpose. Processes $p_{2}$ and $p_{3}$ decide, say 0 ,

8. In a sense, we consider a reduction proof technique similar to that of [22], where the case $n=3$ and $f=1$ is first considered and then generalized. 
$\mathrm{R} 1$

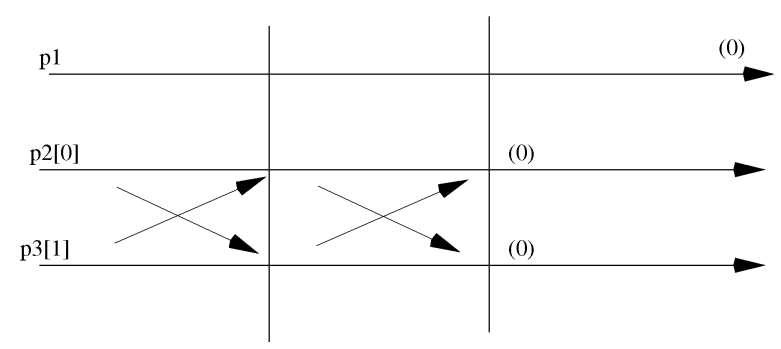

R3

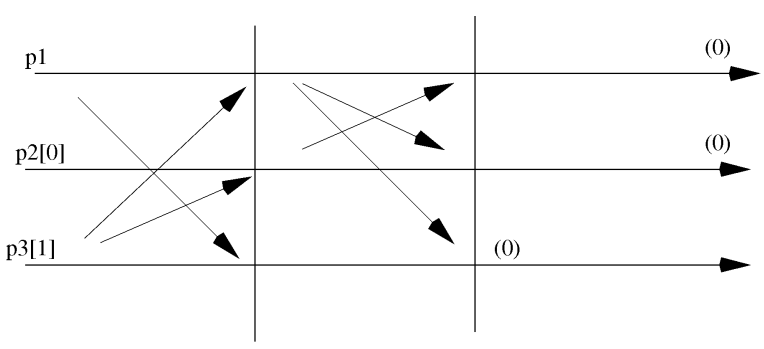

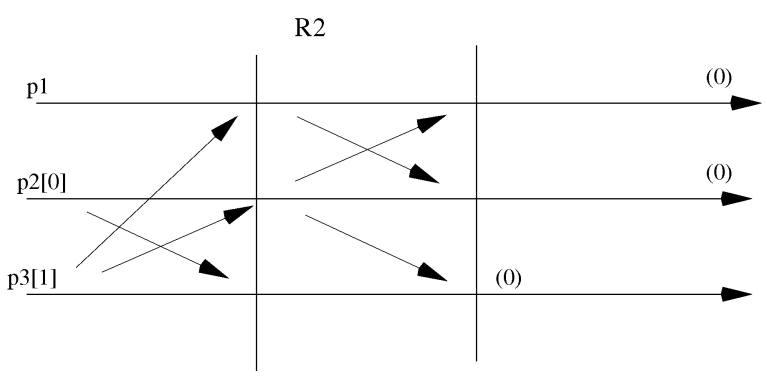

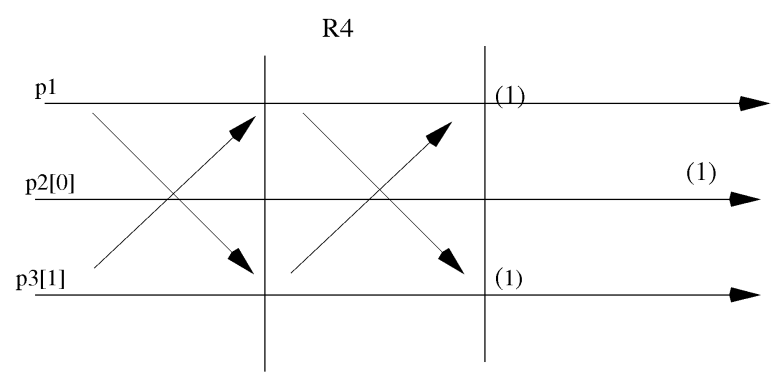

Fig. 8. Runs R1-R4 used in the proof of Theorem 9.

after two rounds (zero-degradation) and, hence, $p_{1}$ eventually decides 0 as well.

2. Process $p_{3}$ cannot distinguish $R_{1}$ from run $R_{2}$ up to the second round: $p_{3}$ receives exactly the same messages from $p_{2}$ and gets the same output from its oracle. Hence, $p_{3}$ decides 0 after two rounds in run $R_{2}$ as well. Processes $p_{1}$ and $p_{2}$ have to eventually decide 0 in $R_{2}$, even if $p_{3}$ crashes immediately after deciding at round 2 .

3. Consider now run $R_{3}$. After two rounds, $p_{1}$ and $p_{2}$ cannot distinguish run $R_{3}$ from $R_{2}$ where $p_{3}$ might have decided 0 after two steps. Assume again that $p_{3}$ crashes immediately after the second round in $R_{3}$. Hence, $p_{1}$ and $p_{2}$ also have to eventually decide 0 in run $R_{3}$ as well. Process $p_{3}$ cannot distinguish run $R_{3}$ from $R_{4}$.

4. In run $R_{4}$, all processes might have the same initial value and if we assume that $A$ is configuration-efficient, then $p_{3}$ must decide 1 after two steps. A contradiction as $p_{3}$ cannot distinguish $R_{4}$ from $R_{3}$.

Case $n>3$. Let us divide the set of processes into three subsets, $P_{1}, P_{2}$, and $P_{3}$, each of size less than or equal to $\lceil n / 3\rceil$. Moreover, let $f$ be such that $n / 2>f \geq\lceil n / 3\rceil$. Given that $f \geq\lceil n / 3\rceil$, all processes of a given subset can crash in a run. The generalization of the proof for any $n$ is then straightforward. We replace process $p_{1}$ with the set of processes $P_{1}$, process $p_{2}$ with set $P_{2}$, and process $p_{3}$ with set $P_{3}$, i.e., if we crash $p_{i}$, we crash all processes in $P_{i}$, if $p_{i}$ proposes a value $v$, we have that value proposed by all processes in $P_{i}$, and so forth. We then follow the same reasoning as for the proof with $n=3$ to construct four runs, as $R_{1}-R_{4}$, and make them contradictory.

Consider now a communication open model. Any message that is sent by a correct process is eventually received by all correct processes. The point here is that, given the asynchronous characteristic of the channels, these messages might arrive after the decision was made. They simply do not change the contradiction argument. $\square$

\subsection{Trading Zero-Degradation}

This section discusses the possibility of trading zerodegradation with configuration-efficiency. The issue is to devise algorithms that are oracle-efficient and configuration-efficient instead of zero-degrading. Recall that oracleefficiency (that concerns crash-free runs) is a weaker property than zero-degradation (that concerns runs with only initial crashes).

\subsubsection{The Case of $\Omega$}

We show below that any $\Omega$-based consensus algorithm that is oracle-efficient is also zero-degrading. As a consequence of our previous impossibility result (Theorem 9), there is no way to trade the zero-degrading characteristic of oracleefficient $\Omega$-based consensus algorithms with configurationefficiency.

Theorem 10. Assuming $f<n / 2$, any oracle-efficient $\Omega$-based consensus algorithm is also zero-degrading.

Proof. Let $A$ be any $\Omega$-based consensus algorithm assuming $f<n / 2$. We need to show that if any run of $A$, where the oracle behaves perfectly and no process crashes, reaches consensus in two steps, then any run of $A$ where the oracle behaves perfectly and no process crashes, except initially, also reaches consensus in 2 steps. Our proof argument is by contradiction.

For presentation simplicity, we simply consider the case of three processes, i.e., $f=1$, in a communicationclosed model and exhibit two contradictory runs, depicted in Fig. 9. $A$ being oracle-efficient, let us assume without loss of generality that 1) $A$ has a run $R_{1}$ where 


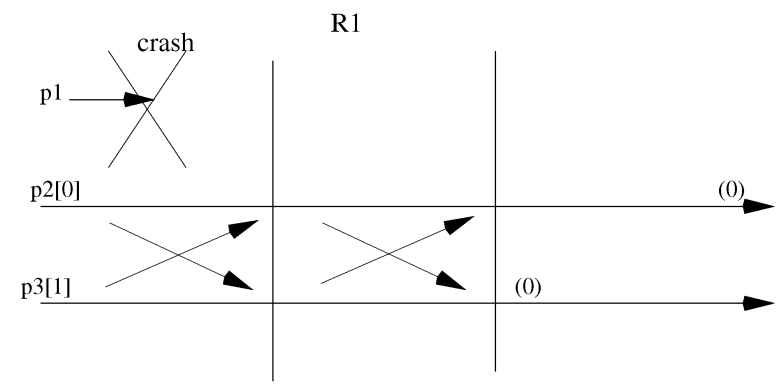

Fig. 9. Runs $R 1-R 2$ used in the proof of Theorem 10.

some process, say $p_{1}$, crashes initially, 2 ) the oracle behaves perfectly, say by permanently electing $p_{2}$, and 3) either $p_{2}$ or $p_{3}$ decides after round 2 . Let us observe that the oracle $\Omega$ might also output $p_{2}$ in a run $R_{2}$ that is similar to $R_{1}$, except for $p_{1}$ that does not crash. Processes $p 2$ and $p_{3}$ cannot distinguish $R_{1}$ from $R_{2}$ as, in both runs, they get the same information from $\Omega$. But then, in $R_{2}, \Omega$ behaves perfectly, no process crashes, and some process decides after round 2: a contradiction as $A$ is an oracle-efficient $\Omega$-based consensus algorithm.

\subsubsection{The Case of $\diamond \mathcal{S}$}

We give here a $\diamond \mathcal{S}$-based consensus algorithm assuming $f<n / 2$ that is oracle-efficient and configuration-efficient. The algorithm is, however, not zero-degrading (this would contradict Theorem 9).

The idea in this $\diamond \mathcal{S}$-based consensus algorithm consists of customizing its first round. More precisely, the lambda () function is implemented as follows:

- $\quad$ Round $r=1$ : The processes exchange their current estimates values and every process waits until it receives a) a majority of estimates and b) an estimate from all nonsuspected processes. If a process 1) receives the same value $v$ or 2 ) receives values from all processes and $v$ is 2.1) the majority among those or 2.2) $p_{1}$ 's value if there is no such majority, then the process returns $v$. Otherwise, the process returns $\perp$. (Let us notice that this first round relies only on the majority of correct processes assumption and the strong completeness property of $\diamond \mathcal{S}$.)

- Round $r>1$ : These rounds use the module described in Fig. 3.

When we consider this implementation of the lambda () function, the first round satisfies validity, quasi-agreement, fixed point, and termination and the other rounds additionally satisfy eventual convergence. Consider a consensus algorithm using this implementation of lambda. Clearly, if all processes propose the same value, the processes return that value within lambda and all correct processes decide in two steps (configuration-efficiency). Similarly, if the oracle behaves perfectly and no process crashes, then all processes get all values and return the same value within lambda: Thus, the processes decide in two steps (oracle-efficiency).

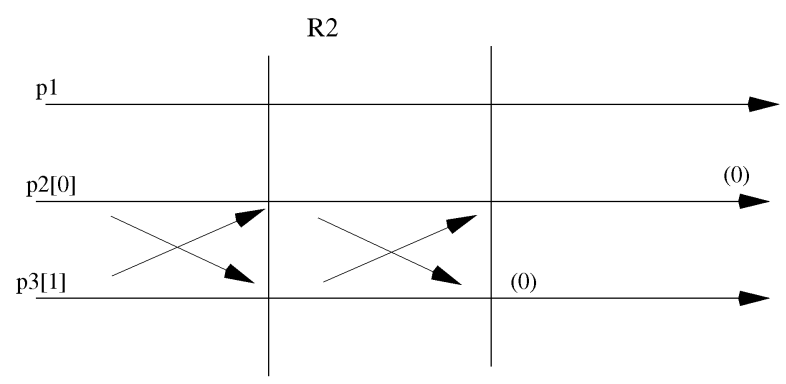

\subsubsection{On Perfect $\Omega$ and $\diamond \mathcal{S}$ Oracles}

While $\Omega$ and $\diamond \mathcal{S}$ have the same computational power [8], [10], it is important to notice that perfect $\Omega$ and perfect $\diamond \mathcal{S}$ are not equivalent. This observation might help get an intuition of why any oracle-efficient $\Omega$-based consensus algorithm is also zero-degrading, which is not the case with $\diamond \mathcal{S}$.

\section{Conclusion}

This paper dissects the information structure of consensus algorithms that rely on oracles such as the random oracle [3], the leader oracle [21], and the failure detector oracle [7]. The algorithms we consider are indulgent toward their oracle: No matter how the underlying oracle behaves, consensus safety is never violated.

We encapsulate the information structure of indulgent consensus algorithms within a new distributed abstraction, called Lambda. Basically, this abstraction is invoked in the first phase of every round of our generic consensus algorithm. It highlights a deep unity in the design principles of consensus solutions and allows to state, in an abstract way, the properties the oracles equipping the underlying asynchronous system have to satisfy. This not only allows us to provide a single proof of a family of algorithms (whatever the oracles effectively used), but also promotes the design of new oracles appropriately defined according to the practical setting in which the system has to run.

The genericity of the approach helps devise new consensus algorithms that are, at the same time, oracleefficient, zero-degrading, and one-step-deciding. We could also derive new lower bounds such as the impossibility of having an algorithm that is zero-degrading and configuration-efficient at the same time.

It is important to notice that our approach does not aim at unifying all algorithmic principles underlying consensus. In particular, we focused on indulgent consensus algorithms [15]. Figuring out how to include, for instance, the synchronous dimension in our general information structure might be feasible along the lines of [14], but requires a careful study. Similarly, we did not consider the memory dimension of consensus algorithms to unify models with crash-stop message passing, crash-recovery message passing, and shared memory [4]. Integrating this dimension to the oracle one is yet another nontrivial challenge.

\section{ACKNOWLEDGMENTS}

The authors are grateful to the referees for their useful comments that helped us improve the presentation of this 
paper. They would also like to thank Partha Dutta, Achour Mostéfaoui, Bastian Pochon, and Sergio Rasjbaum for discussions on consensus algorithms and lower bounds. This paper is a revised and extended version of the paper "A Generic Framework for Indulgent Consensus" which appeared in the Proceedings of the 23rd IEEE International Conference on Distributed Computing Systems (ICDCS '03), May 2003.

\section{REFERENCES}

[1] M.K. Aguilera and S. Toueg, "Failure Detection and Randomization: A Hybrid Approach to Solve Consensus," SIAM J. Computing, vol. 28, no. 3, pp. 890-903, 1998.

[2] H. Attiya and J. Welch, Distributed Computing: Fundamentals, Simulations and Advanced Topics. McGraw-Hill, 1998.

[3] M. Ben-Or, "Another Advantage of Free Choice: Completely Asynchronous Agreement Protocols," Proc. Second ACM Symp. Principles of Distributed Computing (PODC '83), pp. 27-30, 1983.

[4] R. Boichat, P. Dutta, S. Frolund, and R. Guerraoui, "Deconstructing Paxos," SIGACT News, Distributed Computing Column, 2003.

[5] F. Brasileiro, F. Greve, A. Mostefaoui, and M. Raynal, "Consensus in One Communication Step," Proc. Sixth Int'l Conf. Parallel Computing Technologies ( $\mathrm{PaCT}$ '01), pp. 42-50, 2001.

[6] J. Brzezinsky, J.-M. Hélary, M. Raynal, and M. Singhal, “Deadlock Models and a General Algorithm for Distributed Deadlock Detection," J. Parallel and Distributed Computing, vol. 31, no. 2, pp. 112-125, 1995.

[7] T. Chandra and S. Toueg, "Unreliable Failure Detectors for Reliable Distributed Systems," J. ACM, vol. 43, no. 2, pp. 225267, 1996.

[8] T. Chandra, V. Hadzilacos, and S. Toueg, "The Weakest Failure Detector for Solving Consensus," J. ACM, vol. 43, no. 4, pp. 685722, 1996.

[9] B. Chor and L. Nelson, "Solvability in Asynchronous Environments: Finite Interactive Tasks," SIAM J. Computing, vol. 29, no. 2, pp. 351-377, 1999.

[10] F. Chu, "Reducing $\Omega$ to $\diamond W$," Information Processing Letters, vol. 67, no. 6, pp. 289-293, 1998.

[11] P. Dutta and R. Guerraoui, "Fast Indulgent Consensus with Zero Degradation," Proc. Fourth European Dependable Computing Conf. (EDCC '02), pp. 191-208, 2002.

[12] C. Dwork, N. Lynch, and L. Stockmeyer, "Consensus in the Presence of Partial Synchrony," J. ACM, vol. 35, no. 2, pp. 288-323, 1988.

[13] M.J. Fischer, N. Lynch, and M.S. Paterson, "Impossibility of Distributed Consensus with One Faulty Process," J. ACM, vol. 32, no. 2, pp. 374-382, 1985.

[14] E. Gafni, "A Round-by-Round Failure Detector: Unifying Synchrony and Asynchrony," Proc. 17th ACM Symp. Principles of Distributed Computing (PODC '98), pp. 143-152, 1998.

[15] R. Guerraoui, "Indulgent Algorithms," Proc. 19th ACM Symp. Principles of Distributed Computing (PODC '00), pp. 289-298, 2000.

[16] V. Hadzilacos and S. Toueg, "Reliable Broadcast and Related Problems," Distributed Systems, S. Mullender, ed., pp. 97-145, New-York: ACM Press, 1993.

[17] J.-M. Hélary, A. Mostéfaoui, and M. Raynal, "A General Scheme for Token- and Tree-Based Distributed Mutual Exclusion Algorithms," IEEE Trans. Parallel and Distributed Systems, vol. 5, no. 11, pp. 1185-1196, Nov. 1994.

[18] M. Hurfin, A. Mostéfaoui, and M. Raynal, "A Versatile Family of Consensus Protocols Based on Chandra-Toueg's Unreliable Failure Detectors," IEEE Trans. Computers, vol. 51, no. 4, pp, 395-408, Apr. 2002.

[19] I. Keidar and S. Rajsbaum, "On the Cost of Fault-Tolerant Consensus when There Are No Faults: A Tutorial," SIGACT News, Distributed Computing Column, vol. 32, no. 2, pp. 45-63, 2001

[20] A.D. Kshemkalyani and M. Singhal, "On the Characterization and Correctness of Distributed Deadlock Detection," J. Parallel and Distributed Computing, vol. 22, no. 1, pp. 44-59, 1994.

[21] L. Lamport, "The Part-Time Parliament," ACM Trans. Computer Systems, vol. 16, no. 2, pp. 133-169, 1998

[22] L. Lamport, R. Shostak, and L. Pease, "The Byzantine General Problem," ACM Trans. Programming Languages and Systems, vol. 4, no. 3, pp. 382-401, 1982.
[23] N. Lynch, Distributed Algorithms. San Francisco: Morgan Kaufmann, 1996.

[24] A. Mostéfaoui, S. Rajsbaum, and M. Raynal, "Conditions on Input Vectors for Consensus Solvability in Asynchronous Distributed Systems," Proc. 33rd ACM Symp. Theory of Computing (STOC '01), pp. 153-162, 2001

[25] A. Mostéfaoui, S. Rajsbaum, and M. Raynal, "A Versatile and Modular Consensus Protocol," Proc. Int'l IEEE Conf. Dependable Systems E Networks (DSN '02), pp. 364-373, 2002.

[26] A. Mostéfaoui and M. Raynal, "Solving Consensus Using Chandra-Toueg's Unreliable Failure Detectors: A General Quorum-Based Approach," Proc. 13th Int'l Symp. Distributed Computing (DISC'99), pp. 49-63, 1999.

[27] A. Mostéfaoui and M. Raynal, "Leader-Based Consensus," Parallel Processing Letters, vol. 11, no. 1, pp. 95-107, 2001.

[28] A. Mostéfaoui, M. Raynal, and F. Tronel, "The Best of Both Worlds: A Hybrid Approach to Solve Consensus" Proc. Int'l IEEE Conf. Dependable Systems \& Networks (DSN '00), pp. 513-522, 2000.

[29] L. Rodrigues and P. Verissimo, "Topology-Aware Algorithms for Large Scale Communications," Advances in Distributed Systems, pp. 127-156, Springer-Verlag, 2000.

[30] B. Sanders, "The Information Structure of Distributed Mutual Exclusion Algorithms," ACM Trans. Computer Systems, vol. 5, no. 3, pp. 284-299, 1987.

[31] A. Schiper, "Early Consensus in an Asynchronous System with a Weak Failure Detector," Distributed Computing, vol. 10, pp. 149157, 1997.

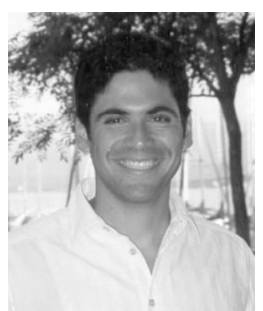

Rachid Guerraoui received the PhD degree in 1992 from the University of Orsay. He has been a professor in computer science since 1999 at EPFL (Ecole Polytechnique Fédérale de Lausanne) in Switzerland, where he founded the distributed programming laboratory. Prior to that, he was with HP Labs in Palo Alto, California, the Center of Atomic Energy (CEA) in Saclay, France, and the Centre de Recherche de l'Ecole des Mines de Paris. His research interests include distributed algorithms and distributed programming languages. In these areas, he has been principal investigator for a number of research grants and has published papers in various journals and conferences. He has served on program committees for various conferences and chaired the program committees of ECOOP 1999, Middleware 2001, and SRDS 2002. He is a member of the IEEE.

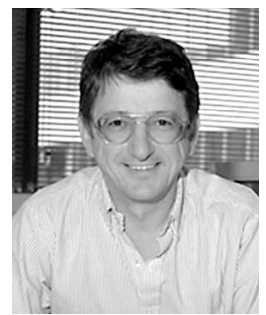

Michel Raynal has been a professor of computer science since 1981. At IRISA (CNRS-INRIAUniversity joint computing research laboratory located in Rennes), he founded a research group on distributed algorithms in 1983. His research interests include distributed algorithms, distributed computing systems, networks, and dependability. His main interest lies in the fundamental principles that underlie the design and the construction of distributed computing systems. He has been principal investigator for a number of research grants in these areas and has been invited by many universities to give lectures about operating systems and distributed algorithms in Europe, South and North America, Asia, and Africa. He serves as an editor for several international journals. He has published more than 75 papers in journals and more than 160 papers in conferences He has also written seven books devoted to parallelism, distributed algorithms, and systems (MIT Press and Wiley). He has served on program committees for more than 70 international conferences and chaired the program committees of more than 15 international conferences. He currently serves as the chair of the steering committee leading the DISC symposium series. He received the IEEE ICDCS best paper Award three times in a row: 1999, 2000, and 2001.

$\triangleright$ For more information on this or any computing topic, please visit our Digital Library at www.computer.org/publications/dlib. 